\title{
Kinetic theory of collective excitations and damping in Bose-Einstein condensed gases
}

\author{
U. Al Khawaja and H. T. C. Stoof \\ Institute for Theoretical Physics, University of Utrecht, Princetonplein 5, 3584 CC Utrecht, The Netherlands
}

(Received 3 April 2000; published 11 October 2000)

\begin{abstract}
We calculate the frequencies and damping rates of the low-lying collective modes of a Bose-Einstein condensed gas at nonzero temperature. We use a complex nonlinear Schrödinger equation to determine the dynamics of the condensate atoms, and couple it to a Boltzmann equation for the noncondensate atoms. In this manner we take into account both collisions between noncondensate-noncondensate and condensatenoncondensate atoms. We solve the linear response of these equations, using a time-dependent Gaussian trial function for the condensate wave function and a truncated power expansion for the deviation function of the thermal cloud. As a result, our calculation turns out to be characterized by two dimensionless parameters proportional to the noncondensate-noncondensate and condensate-noncondensate mean collision times. We find in general quite good agreement with experiment, both for the frequencies and damping of the collective modes.
\end{abstract}

PACS number(s): 03.75.Fi, 03.65.Db, 05.30.Jp, 32.80.Pj

\section{INTRODUCTION}

The nonzero-temperature dependence of the frequencies and damping of collective modes in trapped atomic Bose gases has been investigated extensively both experimentally [1-4] and theoretically [5-24]. Above the Bose-Einstein transition temperature the lowest-lying collective modes have been calculated in the collisionless regime using a collisionless Boltzmann equation or Landau-Vlasov equation with mean-field effects being neglected [17], and in the hydrodynamic regime either by using the conservation laws of hydrodynamics [5] or by using the appropriate quantum kinetic equation [6]. In the collisionless regime the frequency of the collective mode is large compared to the mean collision frequency, in contrast to the situation in the hydrodynamic regime. Several papers have also studied the regime intermediate between the collisionless and hydrodynamic regimes by taking into account interatomic collisions $[17,23,24]$, which turn out to mostly lead to mode damping. These papers have indicated that the experiments were performed under conditions intermediate between collisionless and hydrodynamic. (This is particularly true for the experiments performed by Mewes et al. [3] and Stamper-Kurn et al. [4].) For temperatures far below the transition temperature the collisionless modes can be described accurately by the time-dependent Gross-Pitaevskii equation [25]. At higher temperatures the noncondensate fraction becomes substantial and the modes of the condensate are now coupled to those of the thermal cloud. In Refs. [8,9,11] the temperature dependence of the mode frequencies has been calculated by employing the Popov approximation to include the static meanfield effects of the noncondensate atoms in the GrossPitaevskii equation. This was improved later by taking into account also the dynamics of the thermal cloud using a random phase approximation (RPA) [10] or the collisionless Boltzmann equation [21]. For the hydrodynamic regime a two-fluid model has been developed in Refs. [14,15]. Moreover, the theory of Zaremba, Griffin, and Nikuni [14] was improved later by the same authors to include collisions between condensate and noncondensate atoms [18].

Using a combination of two previous papers, namely
Refs. [21] and [24], we aim in this paper at interpolating between the collisionless and hydrodynamic regimes for experiments below the transition temperature. The authors of Ref. [24] have already performed such an interpolation for a gas above the critical temperature by using a Boltzmann equation with a relaxation time approximation. In contrast, the authors of Ref. [21] use a collisionless Boltzmann equation for the thermal cloud coupled to a time-dependent nonlinear Schrödinger equation for the condensate to consider the collisionless dynamics below the critical temperature. Here, we thus combine these two approaches by using again the Boltzmann equation and time-dependent nonlinear Schrödinger equation, but now including also the effects of interatomic collisions in the manner as put forward in Refs. [14] and [22]. This implies that we have to add two collision terms to the Boltzmann equation. The first collision term represents collisions between two noncondensate atoms and the second describes collisions between a condensate and a noncondensate atom. We use for both these collision terms a relaxation time approximation, since this approximation leads above the transition temperature to a good agreement with microscopic calculations as well as with experimental data [24]. Furthermore, for consistency reasons we also have to include a damping term in the time-dependent nonlinear Schrödinger equation, which is due to collisions between the condensate and noncondensate atoms. As a result our calculation will essentially be characterized by two parameters, namely, $\tau_{22}$ and $\tau_{12}$, denoting the mean collision time for collisions between the noncondensate atoms and between condensate and noncondensate atoms, respectively. Note that we use here the same notation as in Ref. [18]. This will allow us to investigate the collisionless and hydrodynamic limits with respect to both $\tau_{22}$ and $\tau_{12}$, and enable us to fit our results for frequencies and damping with the experimental data.

To solve the complicated nonlinear dynamics of the gas, we employ a Gaussian trial function for the wave function of the condensate atoms with three complex time-dependent variational parameters. For the thermal cloud we use a distribution function that incorporates deviations from the BoseEinstein distribution function. The deviation function is a 
truncated power expansion in the momenta and coordinates of the atoms. Our system of coupled equations is then assembled from the Euler-Lagrange equations for the variational parameters in the condensate wave function and from all the linear and quadratic moments of the Boltzmann equation for the distribution function of the noncondensate atoms. The solution of the linearized version of this system of coupled equations results in a dispersion relation that gives the frequencies and damping of the coupled modes in terms of $\tau_{22}$ and $\tau_{12}$. Although we restrict our calculation to the axially symmetric traps used in the experiments, the generalization to fully anisotropic traps is straightforward.

The rest of this paper is organized as follows. In the next section we present the theoretical details of our calculation. Specifically, we write down the time-dependent nonlinear Schrödinger equation for the condensate wave function and the linearized Boltzmann equation for the thermal cloud, and show how to treat the two collision terms in a relaxation time approximation. In Sec. II A we present our trial functions for the condensate wave function and noncondensate distribution function and in Sec. II B we discuss how we obtain the equilibrium state of the gas around which we have to expand to find the collective modes. In Sec. III we present the resulting dispersion relation and discuss its collisionless and hydrodynamic limits. We then compare our results with experiment. We end in Sec. IV by summing up our main conclusions.

\section{COUPLED DYNAMICS OF THE CONDENSATE AND THE THERMAL CLOUD}

It has been shown that the coupled time-dependent nonlinear Schrödinger equation and the quantum Boltzmann equation can both be derived starting from the equation of motion $[11,18,22]$

$$
i \hbar \frac{\partial \hat{\psi}(\mathbf{r}, t)}{\partial t}=\left(-\frac{\hbar^{2} \nabla^{2}}{2 m}+V^{\text {ext }}(\mathbf{r})+g \hat{\psi}^{\dagger}(\mathbf{r}, t) \hat{\psi}(\mathbf{r}, t)\right) \hat{\psi}(\mathbf{r}, t),
$$

for the Heisenberg field operator $\hat{\psi}(\mathbf{r}, t)$. Here, $g$ is the effective two-body interaction, which is given in terms of the scattering length $a$ and the atomic mass $m$ as $g=4 \pi a \hbar^{2} / m$. The external potential we take here is a harmonic potential of the general form

$$
V^{\mathrm{ext}}(\mathbf{r})=\frac{1}{2} m\left(\omega_{1}^{2} x^{2}+\omega_{2}^{2} y^{2}+\omega_{3}^{2} z^{2}\right),
$$

where $\omega_{i}$ is the characteristic frequency of the trap in the $i$ th direction. To obtain the time-dependent nonlinear Schrödinger equation we write $\hat{\psi}(\mathbf{r}, t)$ as

$$
\hat{\psi}(\mathbf{r}, t)=\Phi(\mathbf{r}, t)+\hat{\psi}^{\prime}(\mathbf{r}, t),
$$

where $\Phi(\mathbf{r}, t)$ is the appropriate nonequilibrium expectation value of $\hat{\psi}(\mathbf{r}, t)$ and the operator $\hat{\psi}^{\prime}(\mathbf{r}, t)$ describes the noncondensate atoms. The number density of condensate atoms $n_{c}(\mathbf{r}, t)$ is related to $\Phi(\mathbf{r}, t)$ by $n_{c}(\mathbf{r}, t)=|\Phi(\mathbf{r}, t)|^{2}$ while the number density of the noncondensate atoms $n^{\prime}$ equals $\left\langle\hat{\psi}^{\prime}(\mathbf{r}, t) \hat{\psi}^{\prime}(\mathbf{r}, t)\right\rangle$, where the brackets represent the averaging over the nonequilibrium density matrix. Substituting this form for $\hat{\psi}(\mathbf{r}, t)$ in the equation of motion and averaging, we obtain

$$
\begin{aligned}
i \hbar \frac{\partial \Phi(\mathbf{r}, t)}{\partial t}= & \left(-\frac{\hbar^{2} \nabla^{2}}{2 m}+V^{\mathrm{ext}}(\mathbf{r})+g n_{c}(\mathbf{r}, t)+2 g n^{\prime}(\mathbf{r}, t)\right. \\
& -i R(\mathbf{r}, t)) \Phi(\mathbf{r}, t)
\end{aligned}
$$

where $R(\mathbf{r}, t)$ is given by

$$
\begin{aligned}
R(\mathbf{r}, t)= & -g\left(\left\langle\hat{\psi}^{\prime}(\mathbf{r}, t) \hat{\psi}^{\prime}(\mathbf{r}, t)\right\rangle \Phi^{*}(\mathbf{r}, t)\right. \\
& \left.+\left\langle\hat{\psi}^{\prime}(\mathbf{r}, t) \hat{\psi}^{\prime}(\mathbf{r}, t) \hat{\psi}^{\prime}(\mathbf{r}, t)\right\rangle\right) / \Phi(\mathbf{r}, t) .
\end{aligned}
$$

Next, the quantum Boltzmann equation can be derived by writing an equation of motion for the distribution function of the noncondensate atoms $f(\mathbf{p}, \mathbf{r}, t)$. This is usually done by writing $f(\mathbf{p}, \mathbf{r}, t)$ as a Wigner transform of $\left\langle\hat{\psi}^{\prime}\left(\mathbf{r}^{\prime}, t\right) \hat{\psi}^{\prime}(\mathbf{r}, t)\right\rangle$ and then determining the time evolution of $f(\mathbf{p}, \mathbf{r}, t)$ from the equation of motion in Eq. (1) $[18,22,26]$. In the Hartree-Fock approximation the resulting Boltzmann equation takes the form

$$
\left[\frac{\partial f}{\partial t}+\boldsymbol{\nabla}_{\mathbf{p}} E \cdot \nabla_{\mathbf{r}}-\nabla_{\mathbf{r}} E \cdot \nabla_{\mathbf{p}}\right] f=C_{22}[f]+C_{12}[f],
$$

where $C_{22}$ is the contribution to the rate of change of $f$ due to collisions between noncondensate atoms, while $C_{12}$ is the contribution due to collisions between the condensate and the noncondensate atoms. The energy $E(\mathbf{p}, \mathbf{r}, t)$ of the noncondensate atoms is in this approximation given by [21]

$$
E(\mathbf{p}, \mathbf{r}, t)=p^{2} / 2 m+V^{e x t}(\mathbf{r})+2 g n(\mathbf{r}, t),
$$

where $n=n_{c}+n^{\prime}$ is the total density.

In Ref. [18] explicit forms of the two collision terms have been written down as follows:

$$
\begin{aligned}
C_{22}[f]= & \frac{2 g^{2}}{(2 \pi)^{5} \hbar^{7}} \int d \mathbf{p}_{2} \int d \mathbf{p}_{3} \int d \mathbf{p}_{4} \delta\left(\mathbf{p}+\mathbf{p}_{2}-\mathbf{p}_{3}-\mathbf{p}_{4}\right) \\
& \times \delta\left(E+E_{2}-E_{3}-E_{4}\right) \\
& \times\left[(1+f)\left(1+f_{2}\right) f_{3} f_{4}-f f_{2}\left(1+f_{3}\right)\left(1+f_{4}\right)\right]
\end{aligned}
$$

and

$$
\begin{aligned}
C_{12}[f]= & \frac{2 g^{2} n_{c}}{(2 \pi)^{2} \hbar^{4}} \int d \mathbf{p}_{1} \int d \mathbf{p}_{2} \int d \mathbf{p}_{3} \\
& \times \delta\left(m \mathbf{v}_{c}+\mathbf{p}_{1}-\mathbf{p}_{2}-\mathbf{p}_{3}\right) \\
& \times \delta\left(E_{c}+E_{1}-E_{2}-E_{3}\right) \\
& \times\left[\delta\left(\mathbf{p}-\mathbf{p}_{1}\right)-\delta\left(\mathbf{p}-\mathbf{p}_{2}\right)-\delta\left(\mathbf{p}-\mathbf{p}_{3}\right)\right] \\
& \times\left[\left(1+f_{1}\right) f_{2} f_{3}-f_{1}\left(1+f_{2}\right)\left(1+f_{3}\right)\right] .
\end{aligned}
$$


Here $E_{c}(\mathbf{r}, t)$ and $\mathbf{v}_{c}(\mathbf{r}, t)$ are the local energy and velocity of the condensate atoms, $E_{i}$ is a shorthand for $E\left(\mathbf{p}_{i}, \mathbf{r}, t\right)$, and similarly $f_{i}$ is a shorthand for $f\left(\mathbf{p}_{i}, \mathbf{r}, t\right)$. As is shown in Ref. [18], the conservation of the total number of atoms relates the collision term $C_{12}$ in the Boltzmann equation to the damping term $R(\mathbf{r}, t)$ in the complex nonlinear Schrödinger equation as

$$
2 n_{c}(\mathbf{r}, t) R(\mathbf{r}, t)=\int \frac{d \mathbf{p}}{(2 \pi)^{3}} C_{12}[f] .
$$

The coupled equations given in Eqs. (4), (6), and (10) in principle fully determine the dynamics of the Bose-Einstein condensed gas in the Hartree-Fock approximation. Since the dynamics of the thermal cloud is experimentally only important for temperatures which are larger or comparable to the mean-field interactions we believe the phonon character of the lowest-lying states in the thermal cloud does not play an important role and we do not need to use the Popov approximation at first instance. Nevertheless, these equations are too complicated to be solved exactly and some approximation is called for.

\section{A. Trial functions}

For the noncondensate atoms we start by linearizing the Boltzmann equation in small deviations of the distribution function around its equilibrium value, namely,

$$
f(\mathbf{p}, \mathbf{r}, t)=f^{(0)}(\mathbf{p}, \mathbf{r})+f^{(0)}(\mathbf{p}, \mathbf{r})\left[1+f^{(0)}(\mathbf{p}, \mathbf{r})\right] \psi(\mathbf{p}, \mathbf{r}, t),
$$

where $f^{(0)}(\mathbf{p}, \mathbf{r})$ is the equilibrium distribution function given by

$$
f^{(0)}(\mathbf{p}, \mathbf{r})=\left(\exp \left\{[E(\mathbf{p}, \mathbf{r})-\mu] / k_{B} T\right\}-1\right)^{-1} .
$$

Here $\mu$ is the chemical potential and $k_{B}$ is Boltzmann's constant. As a result the linearized Boltzmann equation takes the form

$$
\left[\frac{\partial}{\partial t}+\nabla_{\mathbf{p}} E \cdot \nabla_{\mathbf{r}}-\nabla_{\mathbf{r}} E \cdot \nabla_{\mathbf{p}}\right] \psi=C_{22}[\psi]+C_{12}[\psi] .
$$

It is shown in Ref. [24] that for an uncondensed gas a trial function for $\psi(\mathbf{p}, \mathbf{r}, t)$ of the form

$$
\begin{aligned}
\psi= & A_{1} x^{2}+B_{1} x p_{x}+C_{1} p_{x}^{2}+A_{2} y^{2}+B_{2} y p_{y} \\
& +C_{2} p_{y}^{2}+A_{3} z^{2}+B_{3} z p_{z}+C_{3} p_{z}^{2},
\end{aligned}
$$

where $A_{i}(t), B_{i}(t)$, and $C_{i}(t)$ are nine time-dependent functions, is appropriate to describe the low-lying collisionless breathing modes with frequencies $2 \omega_{i}$, some of which have been observed experimentally. We will therefore assume the above expansion to be also reasonably accurate below the critical temperature. For the collision integrals $C_{22}$ and $C_{12}$ we use a relaxation time approximation. In such an approximation one associates these collision integrals to mean relaxation times. However, the approximate expressions for $C_{22}$ and $C_{12}$ should still take into account the conservation laws associated with the collision processes exactly. In the case of $C_{22}$, which represents collisions between the noncondensate atoms, the number of atoms in the thermal cloud, their total momentum, and their total kinetic energy should all be conserved. In our ansatz for $\psi$, terms such as $x^{2}$ and $x p_{x}$ should therefore not be affected by such collisions since they correspond to two collision invariants, namely, the number of atoms and the total momentum in the $x$ direction, respectively. On the other hand, terms such as $p_{x}^{2}$ will be affected by collisions since it is only the sum $p^{2}=p_{x}^{2}+p_{y}^{2}+p_{z}^{2}$ which is conserved during the collision process. Therefore, we can write the following expression for the linear operator $C_{22}$ [20,24]:

$$
C_{22}\left[\psi_{i}\right]=-\frac{1}{\tau_{22}}\left\{\begin{array}{l}
\left(\psi_{i}-p^{2} / 3\right), \quad \psi_{i}=p_{x}^{2}, p_{y}^{2}, p_{z}^{2}, \\
0, \quad \text { otherwise }
\end{array}\right.
$$

where $\tau_{22}$ is a mean collision time for the noncondensatenoncondensate atomic collisions. Note that in this expression $C_{22}\left[p^{2}\right]=0$, ensuring the conservation of the total kinetic energy.

For $C_{12}$, which represents collisions between the condensate and the noncondensate atoms, the number of atoms is not conserved, since the collision process involves transport of atoms back and forth from the condensate into the thermal cloud. This statement means mathematically that the zeroth moment of $C_{12}$, i.e., $\int d \mathbf{p} C_{12}$, does not vanish in contrast to the case of $C_{22}$ where $\int d \mathbf{p} C_{22}=0$. As a first attempt to associate with $C_{12}$ a mean collision time $\tau_{12}$ for noncondensate-condensate atomic collisions, we may write $C_{12} \propto 1 / \tau_{12}$. However, we observe from Eq. (9) that $C_{12}$ $\propto n_{c}(\mathbf{r})$. This dependence on $n_{c}(\mathbf{r})$ requires us to assign to $\tau_{12}$ a position dependence that follows from $n_{c}(\mathbf{r})$ as

$$
\frac{1}{\tau_{12}(\mathbf{r})}=\frac{1}{\tau_{12}(\mathbf{0})} \frac{n_{c}(\mathbf{r})}{n_{c}(\mathbf{0})}
$$

where $\tau_{12}(\mathbf{0})$ and $n_{c}(\mathbf{0})$ are the noncondensate-condensate mean atomic collision time and condensate density at the center of the trap, respectively. Generally, $\tau_{12}(\mathbf{0})$ may, just as $\tau_{22}$, also have temperature dependence. By multiplying and dividing $1 / \tau_{12}(\mathbf{r})$ by $\hbar g$ we can thus take $C_{12}$ as obeying

$$
C_{12} \propto \alpha \frac{2 g n_{c}(\mathbf{r})}{\hbar},
$$

where we introduced the dimensionless constant

$$
\alpha=\frac{\hbar}{2 g n_{c}(\mathbf{0}) \tau_{12}(\mathbf{0})} .
$$

This form for $C_{12}$ is convenient for a reason that becomes clear lateron in this section. It should be emphasized here that the dimensionless parameter $\alpha$ is the second free parameter in our phenomenological calculation. The first dimensionless parameter being $1 / \bar{\omega} \tau_{22}$ where $\bar{\omega}=\sqrt[3]{\omega_{1} \omega_{2} \omega_{3}}$. Therefore, the collisionless and hydrodynamic regimes, as 
well as the intermediate regime, will be described by only these two parameters. Finally, we make the relation between $C_{12}$ and $\alpha$ precise by taking

$$
C_{12}\left[\psi_{i}\right]=-\alpha \frac{2 g n_{c}(\mathbf{r})}{\hbar}\left\{\begin{array}{l}
\left(\psi_{i}-p^{2} / 3\right), \quad \psi_{i}=p_{x}^{2}, p_{y}^{2}, p_{z}^{2}, \\
\psi_{i}, \quad \psi_{i}=x^{2}, y^{2}, z^{2}, \\
0, \quad \text { otherwise. }
\end{array}\right.
$$

This completes our description of the treatment of the thermal cloud. Next we have to consider the condensate.

It is known that a time-dependent Gaussian ansatz for the condensate wave function gives the correct frequencies of the lowest modes at zero temperature [27-29]. Furthermore, the Gaussian ansatz has also been used in Ref. [21] for the whole temperature range below the transition temperature and leads to rather good agreement with experiment. Therefore, we employ here again a Gaussian ansatz for the wave function of the condensate. It has the following form:

$$
\Phi(\mathbf{r}, t)=\sqrt[4]{\frac{8 N_{c} b_{1 r} b_{2 r} b_{3 r}}{\pi^{3}}} \exp \left[-\left(b_{1} x^{2}+b_{2} y^{2}+b_{3} z^{2}\right)\right],
$$

where $b_{1}, b_{2}$, and $b_{3}$ are complex time-dependent variational parameters and $b_{1 r}, b_{2 r}$, and $b_{3 r}$ are their real parts, respectively. Similarly, we denote the imaginary parts, which will appear later on, as $b_{1 i}, b_{2 i}$, and $b_{3 i}$. The prefactor of $\Phi(\mathbf{r}, t)$ guarantees its normalization $\int d \mathbf{r}|\Phi(\mathbf{r}, t)|^{2}$ to be equal to the number of condensate atoms $N_{c}$.

To obtain the first set of our coupled equations of motion, we start by writing down the energy functional that corresponds to the nonlinear Schrödinger equation in Eq. (4), namely,

$E_{c}[\Phi]=\int d \mathbf{r}\left[\frac{\hbar^{2}|\nabla \Phi|^{2}}{2 m}+V^{\mathrm{ext}} n_{c}+\frac{1}{2} g\left(n_{c}^{2}+4 n_{c} n^{\prime}\right)-i R n_{c}\right]$

with $n_{c}=|\Phi|^{2}$. We evaluate this energy functional using the expression for $\Phi$ from Eq. (20), and by noting that the density of the thermal cloud

$$
n^{\prime}=\int \frac{d \mathbf{p}}{(2 \pi)^{3}} f(\mathbf{p}, \mathbf{r}, t)
$$

and the damping term $R(\mathbf{r}, t)$ can be calculated using Eqs. (10) and (19). We notice here that $-i R(\mathbf{r}, t) n_{c}$ turns out to be proportional to the Hartree-Fock interaction term in Eq. (21), with a proportionality constant equals to $-i \alpha$. This explains the reason for writing $C_{12}$ as in Eq. (17). The equations of motion for the condensate dynamics are now the Euler-Lagrange equations resulting from varying the Lagrangian

$$
L=\frac{1}{2} i \hbar \int d \mathbf{r}\left(\Phi^{*} \frac{\partial}{\partial t} \Phi-\Phi \frac{\partial}{\partial t} \Phi^{*}\right)-E_{c}[\Phi]
$$

with respect to the 6 variational parameters $b_{k r}$ and $b_{k i}$.
The second set involves the equations of motion for the constants $A_{i}, B_{i}$, and $C_{i}$. It is obtained from taking appropriate moments of the Boltzmann equation in Eq. (13). In detail, the moments are calculated by multiplying this equation by $f^{(0)}(\mathbf{p}, \mathbf{r})$ and the various components of $\psi$ in Eq. (14), and then integrating over $\mathbf{p}$ and $\mathbf{r}$. This results in nine equations of motion. In combination with the previous 6 ones, we thus have 15 coupled equations of motion. The coupling is provided on the one hand by the mean-field interaction $2 g n_{c} n^{\prime}$ and the imaginary damping term $i R n_{c}$ in the condensate energy functional $E_{c}$ in Eq. (21), and on the other hand, by the contribution $2 g n_{c}$ to the Hartree-Fock energy $E$ in Eq. (7) and the $C_{12}$ collision term in the Boltzmann equation.

\section{B. Equilibrium}

Finally, it is important to note that linearization of the equations of motion of the condensate is obligatory to be consistent with the equations of motion of the noncondensate part which are already linearized. Therefore, we need to calculate the equilibrium state of the gas. In principle, to obtain equilibrium properties we should minimize the free energy $F=E_{\text {tot }}-T S$, where $E_{\text {tot }}$ is the total energy and $S$ is the entropy of the gas, with respect to some variational parameters that characterize the widths of the condensate and the thermal cloud. However, a simplified estimate of the total energy and the entropy contribution to the free energy above the transition temperature shows that for the experimental conditions of interest the former is dominant over the later. This simplified estimate can be made by using a one-parameter Gaussian ansatz for the thermal cloud density, namely $n^{\prime}$ $\propto \exp \left(-x^{2} / R^{2}\right)$, where $R$ is the radius of the cloud. It turns out that in the Thomas-Fermi limit the entropy contribution to the free energy is of order $k_{B} T \ln \gamma^{3 / 5}$, where $\gamma=N^{\prime} a / \bar{a}$, $N^{\prime}$ is the total number of atoms in the thermal cloud, and $\bar{a}=\sqrt{\hbar / m \bar{\omega}}$ is the harmonic oscillator length, while the total energy contribution is of order $k_{B} T \gamma^{2 / 5}$. Therefore, if $\gamma \gg 1$ the entropy contribution is much less than the total energy contribution.

As we shall see in the next section, the experiments were performed with a temperature-dependent total number of atoms ranging from about 6000 atoms at zero temperature to approximately 40000 atoms at the transition temperature. If we extend this simple estimate to temperatures below the transition temperature it turns out that the condition $\gamma \gg 1$ is satisfied only for high temperatures but not for low temperatures since the number of thermal atoms becomes small. However, this condition is then no longer important since the free energy of the thermal cloud at such temperatures is small compared to the condensate energy. Therefore, we will in the following only minimize the total energy $E_{\text {tot }}$ with respect to the variational parameters of the condensate and the thermal cloud. To be able to calculate $E_{\text {tot }}$ we assume that the distribution function of the noncondensate atoms has the same form of that of a noninteracting gas but with varying spatial widths that effectively take into account the meanfield effects of both the noncondensate and condensate atoms. This effective distribution function is written as 


$$
f_{\mathrm{eff}}^{(0)}=\left[\exp \left(\frac{p^{2} / 2 m-\mu}{k_{B} T}+\frac{x^{2}}{R_{1}^{2}}+\frac{y^{2}}{R_{2}^{2}}+\frac{z^{2}}{R_{3}^{2}}\right)-1\right]^{-1},
$$

where $R_{1}, R_{2}$, and $R_{3}$ are the widths of the noncondensate cloud in the three directions. It should be mentioned here that due to the presence of the condensate a "dimple" exists at low temperatures in the noncondensate density profile. As is clear from Eq. (24) we neglect this dimple for two reasons: The first is because we are interested mainly in the hightemperature region where the experiments are performed and the dimple is small. The second reason is that, as argued by Bijlsma and Stoof [21], this dimple produces two competing effects on the frequency shifts. The first effect is to increase the effective mass of the condensate and thus to reduce the mode frequency. The other effect is to lower the mean-field interactions which increases the frequency. The total energy is now a function of six variational parameters

$$
E_{\mathrm{tot}}=E_{\mathrm{tot}}\left(b_{1 r}^{(0)}, b_{2 r}^{(0)}, b_{3 r}^{(0)}, R_{1}, R_{2}, R_{3}\right),
$$

where $b_{1 r}^{(0)}, b_{2 r}^{(0)}$, and $b_{3 r}^{(0)}$ are the equilibrium values of $b_{1 r}$, $b_{2 r}$, and $b_{3 r}$, respectively. The equilibrium is obtained by minimizing this energy with respect to these variational parameters.

The results of such a minimization will be shown in the next section, where we present the dispersion relation that results from solving the above-described system of 15 linearized coupled equations of motion. We discuss also the collisionless and hydrodynamic limits of these results.

\section{THE DISPERSION RELATION: FREQUENCIES AND DAMPING RATES}

Our calculation accounts, in fully anisotropic traps, for nine modes of the gas. In axially symmetric traps this number reduces to six modes. These are the in-phase and out-ofphase combinations of the two monopole $(m=0)$ modes and one quadrupole $(m=2)$ mode of both the condensate and the thermal cloud. Here we denote with $m$ the projection of the angular momentum of the mode along the axis of symmetry of the trap [2]. We focus in this paper on the two lowestlying $m=0$ and $m=2$ modes observed experimentally. It turns out that for the experimentally relevant temperature range, the in-phase $m=0$, and $m=2$ modes correspond mostly to oscillations of the thermal cloud, whereas the outof-phase modes are mostly condensate oscillations. Therefore, we shall often refer in this paper to the in-phase modes as the thermal cloud or noncondensate modes and the out-ofphase modes as the condensate modes. Although our calculation provides results for another, higher-lying, monopole mode, we shall not discuss it further here. Moreover, we emphasize that throughout the following we perform our calculations for parameters taken from the experiments of Jin et al. [2], i.e., with ${ }^{87} \mathrm{Rb}$ atoms in an axially symmetric trap with anisotropy ratio $\omega_{3} / \omega_{1}=\omega_{3} / \omega_{2}=\sqrt{8}$.

In that particular experiment the measurements were performed with a temperature-dependent total number of atoms as a result of the loss of atoms during evaporative cooling. The total number of atoms $N_{\text {tot }}$, as well as the number of

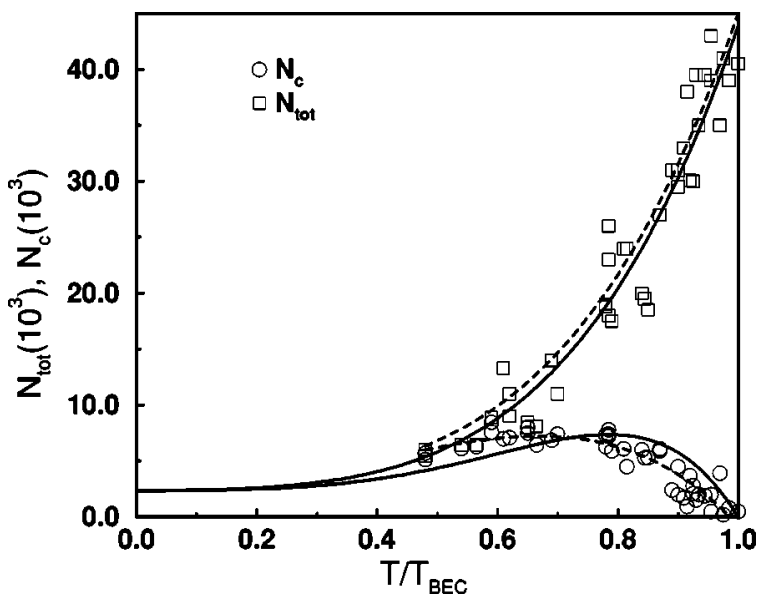

FIG. 1. The measured total number of atoms $N_{\text {tot }}$ (squares) and the number of condensate atoms $N_{c}$ (circles). The dashed lines are a polynomial fit in powers of $T / T_{\mathrm{BEC}}$ to the experimental data. The solid lines represent a fit to $N_{\text {tot }}$ only and using for $N_{c}$ the relation $N_{c}=N_{\text {tot }}\left[1-\left(T / T_{\mathrm{BEC}}\right)^{2}\right]$. The solid line fit of $N_{\text {tot }}$ is shifted slightly downwards in order to get better agreement with the data for $N_{c}$. The measured points were taken from Ref. [2].

condensate atoms $N_{c}$, are measured in the temperature range $T / T_{\mathrm{BEC}} \simeq 0.48$ to $T / T_{\mathrm{BEC}} \simeq 1.0$, where $T_{\mathrm{BEC}}$ is the BoseEinstein transition temperature. These measurements can be easily fitted with polynomials in $T / T_{\mathrm{BEC}}$ as shown by the dashed line in Fig. 1. An extrapolation of such a fit to temperatures below $0.48 T_{\mathrm{BEC}}$ leads, however, to nonphysical situations. In particular, the two curves for $N_{\text {tot }}$ and $N_{c}$ cross at least once before reaching zero temperature. We overcome this problem by fitting only $N_{\text {tot }}$ with the experimental data, and then using $N_{c}=N_{\text {tot }}\left[1-\left(T / T_{B E C}\right)^{2}\right]$ in analogy with the ideal gas relation $N_{c}=N_{\text {tot }}\left[1-\left(T / T_{\mathrm{BEC}}\right)^{3}\right]$ [5]. We note here that we have restricted ourselves to fit the data with a formula that is similar to the ideal gas formula but with a different exponent for the temperature dependence that is regarded as our fitting parameter. We find that an exponent equal to 2 gives the best fit. Although the precise value of the exponent is not obvious to us, the fact that it is less than 3 is physically reasonable since interactions tend to deplete the condensate. Figure 1 shows with the solid line the results of this slightly less accurate fit. It should be noted that using the ideal gas relation will grossly overestimate all the experimental points of $N_{c}$ for $T / T_{\mathrm{BEC}}>0.7$. It turns out that the difference between the calculated frequencies using the less accurate fit from those calculated using the best fit are much smaller than the uncertainties in the measured frequencies for the experimental range of temperatures. This is shown in Fig. 2. Since we want to show also results for the complete temperature interval from zero to $T_{\mathrm{BEC}}$, we employ from now on always the former fit, i.e., the solid lines in Fig. 1.

Before starting with calculating the frequencies and damping rates of the collective modes, we show in Fig. 3 the result of the minimization of the total energy that is required to obtain the equilibrium conditions of the gas, as described in the previous section. We plot the equilibrium widths of both condensate and noncondensate clouds as a function of temperature. We notice from this figure that at zero tempera- 


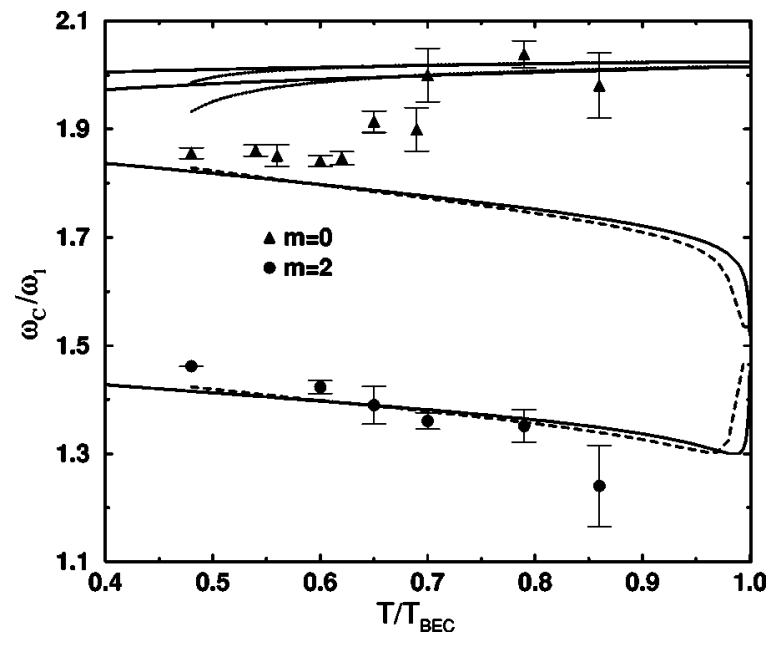

FIG. 2. Collisionless frequencies for $\alpha=0$, i.e., with no collisions between condensate and noncondensate atoms. The solid lines correspond to the approximate fit to the total and condensate number of atoms as shown by the solid lines in Fig. 1. The dashed lines correspond to our best fit in the temperature interval as is given by the dashed lines in Fig. 1. The points are the experimental measurements for the $m=0$ and $m=2$ condensate modes [2]. The labels of the theoretical curves in terms of the values of $m$ are shown in Fig. 4.

ture and at the transition temperature we obtain rather good estimates for the widths of the condensate and thermal clouds. At zero temperature, where the thermal cloud is absent, we observe that the radial width of the condensate equals approximately $2 a_{1}$, a result that is consistent with the familiar property that due to the mean-field interactions the equilibrium condensate width is larger than the ideal gas result $a_{1}$. The axial width, which is roughly $1 / \lambda^{1 / 2}$ of the radial width, is suppressed due to the anisotropy of the trap.

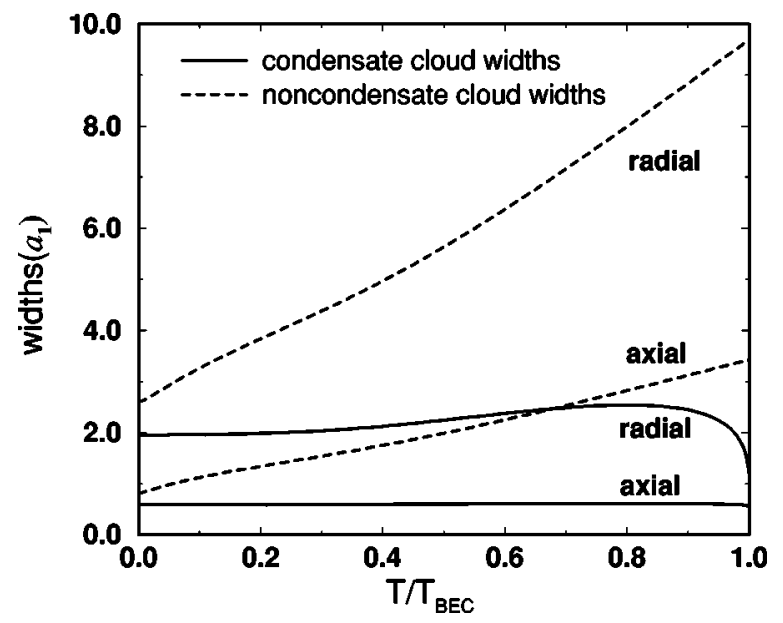

FIG. 3. The equilibrium widths of the condensate and noncondensate clouds in the axially symmetric trap of Ref. [2]. In reference to the text, the radial width of the noncondensate cloud is $R_{1}\left(=R_{2}\right)$, and the axial width is $R_{3}$. For the condensate cloud, the radial width is $\left(2 b_{1 r}^{(0)}\right)^{-1 / 2}\left[=\left(2 b_{2 r}^{(0)}\right)^{-1 / 2}\right]$ and the axial width is $\left(2 b_{3 r}^{(0)}\right)^{-1 / 2}$, as can be seen from Eq. (25). All lengths here are scaled to the harmonic oscillator length $a_{1}=\left(\hbar / m \omega_{1}\right)^{1 / 2}$.
We can also see in this figure that at the transition temperature the radial width of the condensate is slightly larger than the ideal gas one. This slight expansion is caused by the presence of the thermal cloud, whose mean-field interaction has the effect of slightly reducing the spring constants of the effective trapping potential. Finally, we notice that at all temperatures the thermal widths are larger than the condensate ones, indicating that the condensate mean-field expels out the thermal cloud.

Returning to the problem of the collective mode frequencies and damping, we start by neglecting collisions between the condensate and noncondensate atoms, which means that $\alpha=0$. In this case the dispersion relation turns out to have the following general structure:

$$
i \omega\left[P_{C}^{(0)}\left(\omega \tau_{22}\right)^{2}+i P_{I}^{(0)} \omega \tau_{22}-P_{H}^{(0)}\right]=0,
$$

where $P_{C}^{(0)}, P_{I}^{(0)}$, and $P_{H}^{(0)}$ are sixth order polynomials in $\omega^{2}$ which can all be factorized as

$$
\begin{aligned}
& P_{C}^{(0)}=\prod_{k=1}^{6}\left(\omega^{2}-\omega_{C k}^{2}\right), \\
& P_{I}^{(0)}=\prod_{k=1}^{6}\left(\omega^{2}-\omega_{I k}^{2}\right),
\end{aligned}
$$

and

$$
P_{H}^{(0)}=\prod_{k=1}^{6}\left(\omega^{2}-\omega_{H k}^{2}\right) .
$$

Here, $\omega_{C k}, \omega_{H k}$, and $\omega_{I k}, k=1,2,3$, are temperaturedependent collisionless, intermediate, and hydrodynamic frequencies, respectively. The superscripts in $P_{C}^{(0)}, P_{I}^{(0)}$, and $P_{H}^{(0)}$ indicate the value of $\alpha$. The general structure of this dispersion relation agrees with the result of Refs. [23,24] above the transition temperature, where this dispersion relation was studied in detail. Particularly, it was shown that damping rates calculated using this relation agree in order of magnitude with experiments and numerical calculations.

The collisionless regime is defined by the condition $\omega \tau_{22} \gg 1$. Therefore, we find from Eq. (26) that the collisionless frequencies are $\omega_{C k}$. In the hydrodynamic limit $\omega \tau_{22}$ $\ll 1$, and the hydrodynamic frequencies are $\omega_{H k}$. Note that we use the word hydrodynamic here to denote that the thermal cloud is in the hydrodynamic regime though $\alpha=0$ and there are therefore no collisions between condensate and noncondensate atoms. In a sense this regime is thus precisely the limit discussed by Nikuni, Zaremba, and Griffin [18]. Using the experimental parameters we calculate these frequencies for the whole temperature range below $T_{\mathrm{BEC}}$. In Fig. 4 we present the results of this calculation for the $m$ $=0$ and $m=2$ modes together with the experimental data. In this figure the collisionless curves agree with those of $\mathrm{Bi}$ jlsma and Stoof [21] for $T / T_{\mathrm{BEC}}>0.2$, which is not surprising since we use similar ansatz functions. The discrepancy for $T / T_{\mathrm{BEC}}<0.2$ is due to the different ways of treating the equilibrium state. 


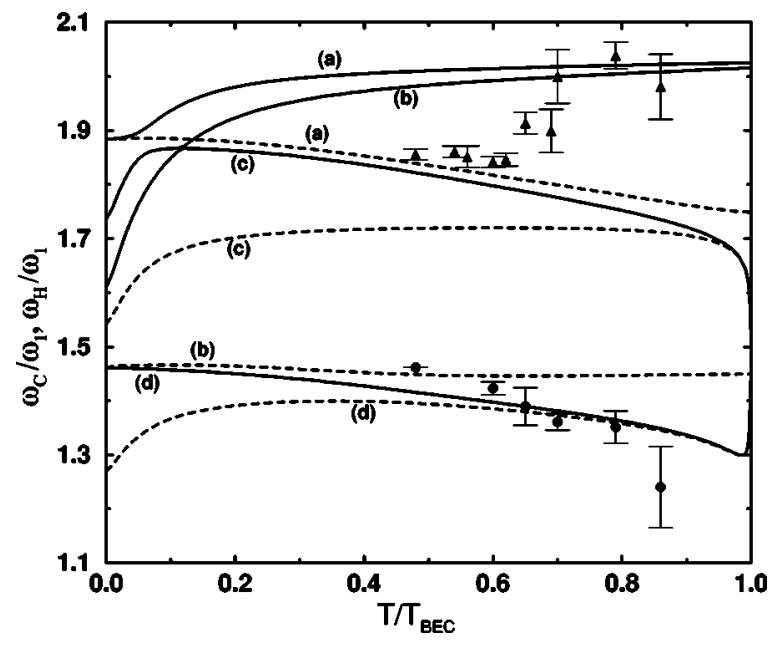

FIG. 4. Collisionless (solid curves) and hydrodynamic (dashed curves) collective mode frequencies for $\alpha=0$. The curves are labeled in terms of the values of $m$ and the phase difference between the condensate and thermal oscillations $\theta$ : (a) $m=0, \theta=0$, (b) $m$ $=2, \theta=0$, (c) $m=0, \theta=\pi$, (d) $m=2, \theta=\pi$.

Above the transition temperature, the explanation of the mode damping was based on the fact that the measured frequencies were less than the theoretical collsionless frequencies. This shift in frequency is then interpreted to be due to the fact that the system is shifted from the collisionless regime towards the hydrodynamic regime. Collisional damping associated with this shift was calculated and compared to the experimental damping. It is clear from Fig. 4 that below the transition temperature this kind of explanation is not possible, since most of the experimental points are not located between the collisionless and hydrodynamic curves.

To investigate the possibility that this damping might be due to the condensate-noncondensate atomic collisions, we now study the effect of these collisions by taking $\alpha$ to be nonzero. For nonzero $\alpha$ the dispersion relation takes the general form

$$
\begin{gathered}
\left(\sum_{k=0}^{9} P_{C}^{(k)} \alpha^{k}\right)\left(\omega \tau_{22}\right)^{2}+i\left(\sum_{k=0}^{8} P_{I}^{(k)} \alpha^{k}\right) \omega \tau_{22} \\
-\sum_{k=0}^{7} P_{H}^{(k)} \alpha^{k}=0 .
\end{gathered}
$$

We note that the $k=0$ term in this expression corresponds to Eq. (26), where $\alpha$ was indeed taken to be zero. Frequencies and damping rates can now be obtained by writing $\omega$ in terms of a real and imaginary parts, $\omega=\omega_{r}+i \omega_{i}$, and then inserting this expression in the last equation. In general, one obtains expressions for $\omega_{r}$ and $\omega_{i}$ in terms of $\bar{\omega} \tau_{22}$ and $\alpha$.

We start our discussion of the frequencies and damping rates for $T / T_{\mathrm{BEC}}=0.79$, since at this temperature the experiment provides data for both the condensate and noncondensate oscillations. We present the results of our calculation for the $m=0$ and $m=2$ modes of the thermal cloud in Fig. 5, where we have eliminated $\bar{\omega} \tau_{22}$ and plotted $\omega_{i}$ as a function of $\omega_{r}$ for $\alpha=0$ and $\alpha=0.25$. On the same plot we show the

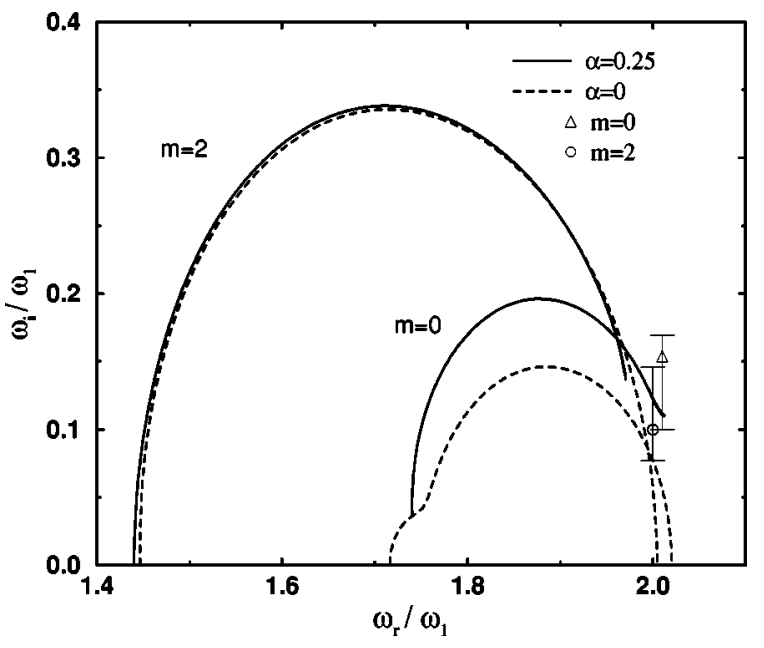

FIG. 5. Damping rate $\omega_{i}$ versus frequency $\omega_{r}$ for noncondensate oscillations, at $T=0.79 T_{\mathrm{BEC}}$ for $\alpha=0$ and $\alpha=0.25$. The two points correspond to the experimentally observed frequency and damping rate of the thermal cloud oscillation.

two experimental points corresponding to the $m=0$ and $m$ $=2$ modes of the noncondensate oscillation. In this figure, the right end of the curves represent the collisionless regime $\left(\bar{\omega} \tau_{22} \gg 1\right)$ and the left end is the hydrodynamic regime $\left(\bar{\omega} \tau_{22} \ll 1\right)$. Therefore, the location where the dashed curves intersect with the horizontal axis can also be seen in Fig. 4 at $T=0.79 T_{\mathrm{BEC}}$. For the experimental point corresponding to the monopole mode, the error bars do not intersect with the dashed curve $(\alpha=0)$, but they do with the solid curve $(\alpha$ $=0.25$ ) indicating that a nonzero value of $\alpha$ is needed to account for the experimental damping. This indicates that condensate-noncondensate atomic collisions are mainly responsible for the observed damping of this mode. This is less clear for the quadrupole mode although the experimental data is certainly not inconsistent with this conclusion.

Next we show in Fig. 6 at the same temperature both the

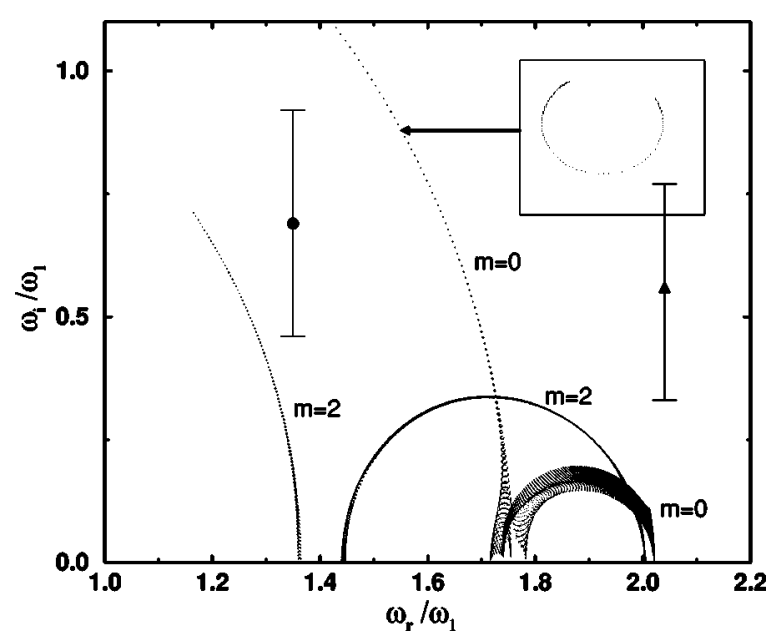

FIG. 6. Damping rate $\omega_{i}$ versus frequency $\omega_{r}$ of condensate and noncondensate oscillations at $T=0.79 T_{\mathrm{BEC}}$, for $\alpha$ changing from 0 to 0.25 in steps of 0.025 . The two points correspond to the experimental measurements of the condensate oscillation. 
condensate as well as the noncondensate $m=0$ and $m=2$ modes for values of $\alpha$ ranging from 0 to 0.25 . The effect of $\alpha$ on the noncondensate modes is a slight shift and rotation of the curves but their general structure is roughly preserved. This can be seen more clearly in Fig. 5. The effect of $\alpha$ on the condensate modes is much larger, it gives rise to a large upward shift for the whole curve. The effect of $\bar{\omega} \tau_{22}$ on these curves becomes smaller for larger values of $\alpha$, which can be seen by noting that the radius of the small semicircles becomes smaller for larger values of $\alpha$. Again, we see that a nonzero value of $\alpha$ provides sufficient damping to account for the experimental observations. However, there is a discrepancy between our calculation and the experiment in the value of the frequency of the $m=0$ mode, since our calculation predicts $\omega / \omega_{1} \approx 1.68 \pm 0.4$, whereas the experimental data give a frequency of about $2.05 \omega_{1}$. This discrepancy was also present in the calculations of Refs. $[10,21]$ and is discussed in Ref. [21]. The authors of the last reference suggested that a possible reason for this discrepancy may be that the observed mode is in fact an in-phase $m=0$ mode (the upper solid curve in Fig. 4) rather than an out-of-phase one. This suggestion was based on calculating the oscillator strengths of the in-phase and out-of-phase $m=0$ modes. It turned out that it is indeed possible experimentally to excite both modes simultaneously in the temperature range $T$ $\approx 0.25 T_{\mathrm{BEC}}$ to $T \approx 0.5 T_{\mathrm{BEC}}$. Although the calculation in Ref. [21] was performed in the collisionless limit, where there is no damping, we expect that with damping this argument remains qualitatively correct.

For the rest of the experimental points we can, in principle, explain the data using a two-parameter fit, namely, with $\alpha$ and $\bar{\omega} \tau_{22}$. In fact, the collisions between atoms from the condensate with those from the thermal cloud are the main cause of damping in the condensate modes, and we can even explain the data using a one-parameter fit, namely, only $\alpha$, by assuming that the system is in the collisionless regime with respect to $\bar{\omega} \tau_{22}$, i.e., $\bar{\omega} \tau_{22} \gg 1$. We perform this calculation by using a function $\alpha\left(T / T_{\mathrm{BEC}}\right)$ that gives the best fit for the experimental damping rates. In Fig. 7 we plot the function $\alpha\left(T / T_{\mathrm{BEC}}\right)$ and the resulting temperature-dependent frequencies and damping rates. This figure contains the main results of this paper. First of all we notice that now, with a nonzero $\alpha$, we obtain better agreement with the experimental data for the quadrupole mode frequencies than before where $\alpha$ was taken to be zero. This can be seen by comparing this figure with Fig. 4. We note also that, depending on the value of $\alpha$, the mode frequency may shift upwards or downwards. By comparing Fig. 4 with Fig. 7 for the mode frequencies one can clearly see that for temperatures below approximately $0.6 T_{\mathrm{BEC}}$ the theoretical curves are shifted upwards, whereas for the higher temperatures the curves are shifted downwards, in the end giving rise to the good agreement of the quadrupole mode with experiment. Secondly, we notice that the same function $\alpha\left(T / T_{\mathrm{BEC}}\right)$ also gives a good fit for the damping rates of both the monopole and quadrupole modes. There is still a discrepancy between our predictions and the experimental findings for the frequencies of the monopole mode at higher temperatures, but this may be re-

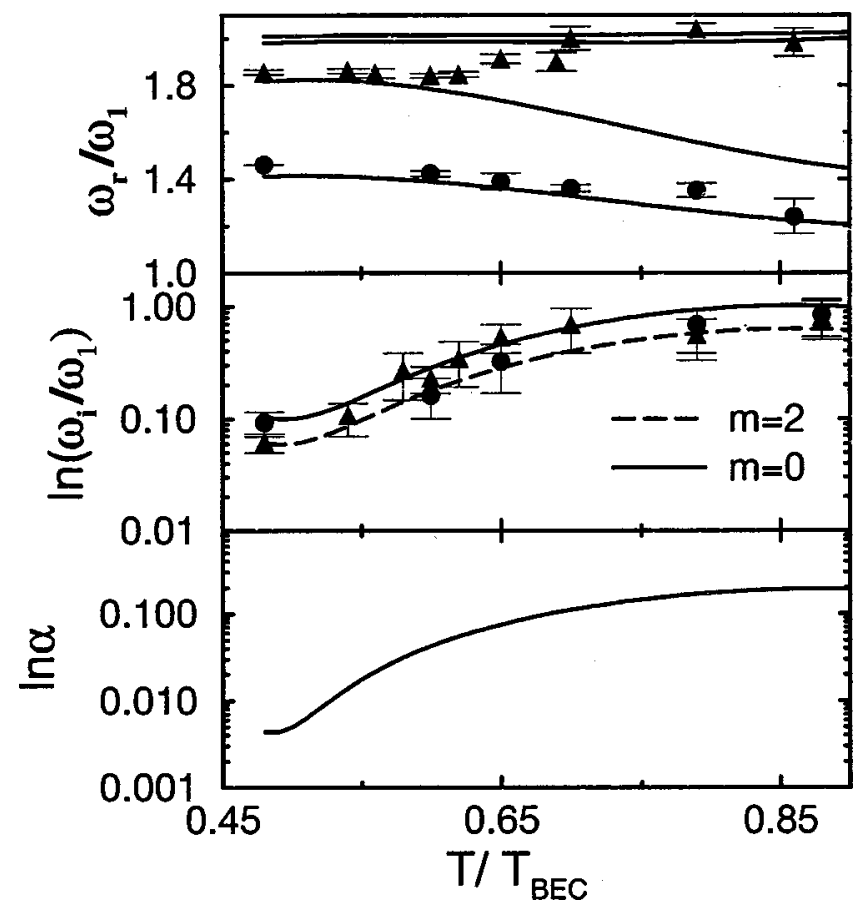

FIG. 7. Fitting all the experimental data on the mode frequencies and damping rates with a single temperature-dependent function $\alpha$. At low temperature the saturation in the curve for $\alpha$ is an artifact of the fitting.

solved by a reinterpretation of the observed mode, as explained previously. Finally, the temperature behavior of $\alpha$ in Fig. 7 indicate that $\alpha$ obeys a power law in temperature. However, an accurate estimate of the exponent is lacking due to the large uncertainties in the experimental data.

Finally, we want to mention an interesting feature of the in-phase and out-of-phase $m=0$ modes which can already be seen in Fig. 4 at $T / T_{\mathrm{BEC}} \approx 0.05$. It is the familiar anticrossing tendency of these modes obtained also by Bijlsma and Stoof [21] and Zaremba et al. [18]. It is more dramatic here than in these two papers due to the presence of the new parameter $\alpha$. This behavior can be seen in Fig. 8 where we see the two modes come close to each other and eventually intersect at some "critical" values of $\alpha$ twice.

\section{CONCLUSION}

We have extended the method of calculating collisional damping, used previously above the Bose-Einstein condensation transition temperature $[23,24]$, to below the transition temperature. Furthermore, we have included the effect of noncondensate-condensate collisions. By comparing with experiment, we conclude that it is presumably this collision process which is mainly responsible for the observed damping. Our theory provides a general dispersion relation that gives complex mode frequencies at a certain temperature as a function of two dimensionless parameters, namely, $1 / \bar{\omega} \tau_{22}$ and $\alpha$, that characterize the noncondensate-noncondensate and noncondensate-condensate atomic collisions, respectively. Our results for the fully collisionless frequencies agree with those of Ref. [21] for most of the temperature 


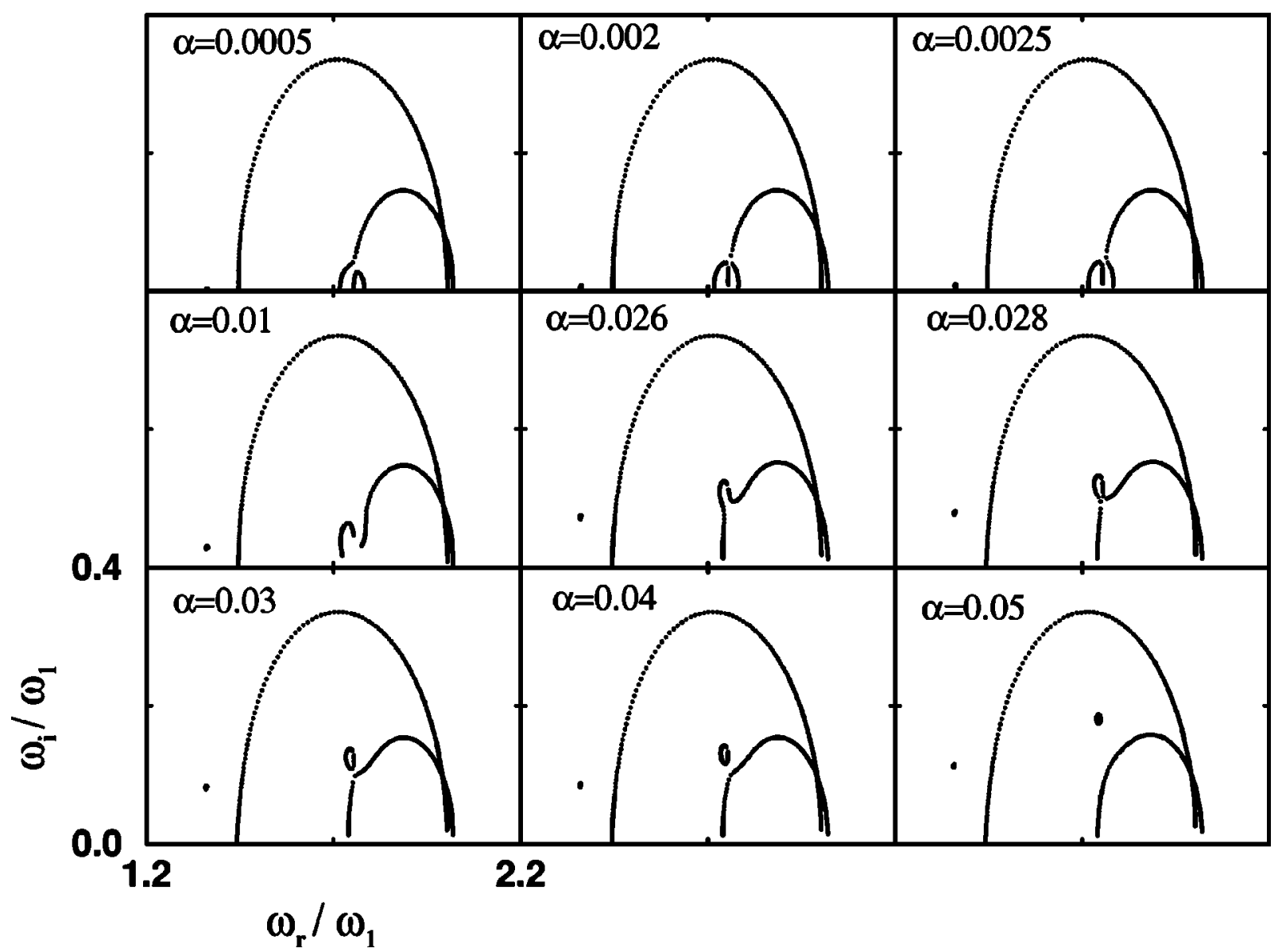

FIG. 8. Damping rate $\omega_{i}$ versus frequency $\omega_{r}$ of condensate and noncondensate oscillations at $T=0.79 T_{\mathrm{BEC}}$ with $\alpha$ changing from 0.0005 to 0.05 . The four curves in each plot correspond to the in-phase and out-of-phase $m=0$ and $m=2$ modes which are also shown in Fig. 6. The present figure corresponds to the region in Fig. 6 where the in-phase and out-of-phase $m=0$ modes come close to each other. One can see here that the anticrossing feature of these two $m=0$ modes is not only present at $\alpha=0$ [21] but also for nonzero values of $\alpha$.

range below the transition temperature. The $m=0$ and $m$ $=2$ hydrodynamic frequencies are, to the best of our knowledge, calculated here below the transition temperature for the first time.

At present we have not carried out a microscopic calculation of the mean collision times $\tau_{22}$ and $\tau_{12} \propto \alpha^{-1}$, which are treated as phenomenological parameters here with the possibility to investigate the intermediate regime with respect to either of the two collision processes. However, the homogeneous calculations of Zaremba, Nikuni, and Griffin [18] indicate that the function $\alpha\left(T / T_{\mathrm{BEC}}\right)$ that we have obtained from a fit to the experimental data, has the correct order of magnitude and qualitatively also the correct temperature dependence. Approaching the transition temperature from below, the microscopic calculation of the latter work gives rise to a divergent mean collision rate. It can be argued that the source of this divergency is at least partially due to the use of a two-body $T$ matrix instead of the many-body $T$ matrix for the binary collision potential and therefore that the latter should be used for an accurate calculation, which however, becomes much more involved. Nevertheless such a mi- croscopic calculation for the inhomogeneous experimental conditions of interest would be very desirable and is left for future work.

We obtain a rather good agreement with the experimental results for the mode frequencies and damping rates apart from the discrepancy for the $m=0$ out-of-phase mode for $T>0.7 T_{\text {BEC }}$. While the experiment shows an upward shift in the frequency with respect to its zero temperature value, we predict a downward shift. Resolution of this discrepancy seems to require more accurate experimental data, which will also provide decisive comparison with the theory presented here.

\section{ACKNOWLEDGMENTS}

The authors would like to thank Michiel Bijlsma, Chris Pethick, and Henrik Smith for useful suggestions and remarks. This work is supported by the Stichting Fundamenteel voor Onderzoek der Materie (FOM), which is financially supported by the Nederlandse Organisatie voor Wetenschappeliijk Onderzoek (NWO). 
[1] D. S. Jin, J. R. Ensher, M. R. Matthews, C. E. Wieman, and E. A. Cornell, Phys. Rev. Lett. 77, 420 (1996).

[2] D. S. Jin, J. R. Ensher, M. R. Matthews, C. E. Wieman, and E. A. Cornell, Phys. Rev. Lett. 78, 764 (1997).

[3] M.-O. Mewes, M. R. Andrews, N. J. van Druten, D. M. Kurn, D. S. Durfee, C. G. Townsend, and W. Ketterle, Phys. Rev. Lett. 77, 988 (1996).

[4] D. M. Stamper-Kurn, H.-J. Miesner, S. Inouye, M. R. Andrews, and W. Ketterle, Phys. Rev. Lett. 81, 500 (1998).

[5] C. J. Pethick and H. Smith, Nordita Lecture Notes.

[6] A. Griffin, W. C. Wu, and S. Stringari, Phys. Rev. Lett. 78, 1838 (1997).

[7] Y. Kagan, E. L. Surkov, and G. Shlyapnikov, Phys. Rev. Lett. 79, 2604 (1997).

[8] D. A. W. Hutchinson, E. Zaremba, and A. Griffin, Phys. Rev. Lett. 78, 1842 (1997).

[9] R. J. Dodd, M. Edwards, C. W. Clark, and K. Burnett, Phys. Rev. A 57, 32 (1998).

[10] S. Giorgini, Phys. Rev. A 57, 2949 (1998).

[11] D. A. W. Hutchinson, R. J. Dodd, and K. Burnett, Phys. Rev. Lett. 81, 2198 (1998).

[12] T. Nikuni and A. Griffin, J. Low Temp. Phys. 111, 793 (1998).

[13] R. J. Dodd, M. Edwards, K. Burnett, and C. Clark, Phys. Rev. A 57, R32 (1998).

[14] E. Zaremba, A. Griffin, and T. Nikuni, Phys. Rev. A 57, 4695 (1998).

[15] V. Shenoy and T.-L. Ho, Phys. Rev. Lett. 80, 3895 (1998).
[16] M. Kavoulakis, C. J. Pethick, and H. Smith, Phys. Rev. A 57, 2938 (1998).

[17] G. M. Kavoulakis, C. J. Pethick, and H. Smith, Phys. Rev. Lett. 81, 4036 (1998).

[18] E. Zaremba, T. Nikuni, and A. Griffin, J. Low Temp. Phys. 116, 277 (1999); T. Nikuni, E. Zaremba, and A. Griffin, Phys. Rev. Lett. 83, 10 (1999).

[19] T. Nikuni, E. A. Griffin, and E. Zaremba, cond-mat/9911336 (unpublished).

[20] G. M. Kavoulakis, C. J. Pethick, and H. Smith, Phys. Rev. A 61, 053603 (2000).

[21] M. J. Bijlsma and H. T. C. Stoof, Phys. Rev. A 60, 3973 (1999).

[22] H. T. C. Stoof, J. Low Temp. Phys. 114, 11 (1999).

[23] D. Guéry-Odelin, F. Zambelli, J. Dalibard, and S. Stringari, Phys. Rev. A 60, 4851 (1999).

[24] U. Al Khawaja, C. J. Pethick, and H. Smith, J. Low Temp. Phys. 118, 127 (2000).

[25] See S. Giorgini, L. P. Pitaevskii, and S. Stringari, Rev. Mod. Phys. 71, 463 (1999), and references therein.

[26] T. R. Kirkpatrick and J. R. Dorfman, Phys. Rev. A 28, 2576 (1983); J. Low Temp. Phys. 58, 308 (1985); 58, 399 (1985).

[27] V. M. Pérez-Garcia, H. Michinel, J. I. Cirac, M. Lewenstein, and P. Zoller, Phys. Rev. Lett. 77, 5320 (1996).

[28] Y. Castin and R. Dum, Phys. Rev. Lett. 77, 5315 (1996).

[29] H. T. C. Stoof, J. Stat. Phys. 87, 1353 (1997). 